\title{
Conducta alimentaria durante el confinamiento por COVID-19 (CoV-Eat Project): protocolo de un estudio transversal en países de habla hispana
}

\author{
Eating behavior during COVID-19 confinement (CoV-Eat Project): Cross- \\ sectional study protocol in Spanish-speaking countries \\ Comportamento alimentar durante o confinamento por COVID-19 (CoV-Eat Project): \\ protocolo de um estudo transversal em países de língua espanhola
}

\author{
Rafael Almendra-Pegueros ${ }^{1,2}$, Eduard Baladia1*, Catalina Ramírez-Contreras ${ }^{3,4}$, Pía Rojas-Cárdenas', \\ Anna Vila-Martí5, José Moya Osorio ${ }^{6}$, Evelia Apolinar-Jiménez ${ }^{7}$, Alberto Lazzara-López'1, \\ Kristian Buhring-Bonacich', María Celeste Nessier', Sophia Eugenia Martínez-Vázquez', Saby Camacho-López',10,

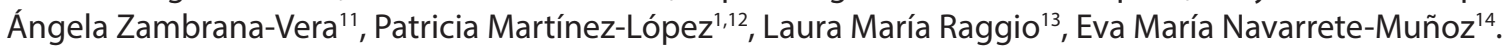

Recibido: 31 de diciembre de 2020. Aceptado para publicación: 9 de enero de 2021.

Publicado en línea: 12 de enero de 2021.

https://doi.org/10.35454/rncm.v4n3.267

\section{Resumen}

Introducción: en diciembre de 2019 surgieron casos de neumonía de etiología desconocida, que más tarde fueron clasificados como coronavirus del síndrome respiratorio agudo grave de tipo 2 (SARS-CoV-2). El 11 de marzo se declaró la pandemia por la enfermedad respiratoria coronavirus disease 2019 (COVID-19), por lo que se recurrió a medidas de contención de la transmisión, como el distanciamiento social y confinamiento, medidas con conocidos efectos estresores que pueden influir en la conducta alimentaria. Por ello, el objetivo de esta investigación es identificar la conducta alimentaria a través de la restricción dietética, alimentación emocional e ingesta incontrolada, y explorar los factores asociados con estas conductas en adultos durante el confinamiento por COVID-19 en países hispanohablantes.

Método: se realizará un estudio transversal descriptivo, mediante un cuestionario en línea autoaplicado, el cual estará integrado por un cuestionario de conducta alimentaria (Three-Factor Eating Questionnaire-R18), información sociodemográfica y de salud, y características del

\section{Summary}

Introduction: In December 2019, cases of pneumonia of unknown etiology emerged, which were later classified as severe acute respiratory syndrome coronavirus 2 (SARS-CoV-2). On March $11^{\text {st }}$, the pandemic by COVID-19 disease was declared, and since then it has been used transmission containment measures such as social distancing and confinement, measures with known effects as stressors that can influence eating behavior. Therefore, the aim of this research protocol is to identify eating behavior through dietary restriction, emotional feeding, and uncontrolled intake and to explore the factors associated with these adult behaviors during the COVID-19 pandemic in Spanish-speaking countries.

Method: A cross-sectional study will be carried out, with a self-applied online questionnaire, which will consist of ThreeFactor Eating Questionnaire-R18, sociodemographic and health information, and lifestyle characteristics before and during confinement. Descriptive analysis of variables, bivariate analyses and multivariate linear regression models will be performed

\section{Resumo}

Introdução: em dezembro de 2019 surgiram casos de pneumonia de etiologia desconhecida, posteriormente classificados como coronavírus de síndrome respiratória aguda grave do tipo 2 (SARSCoV-2). No dia 11 de março, foi declarada a pandemia pela doença respiratória coronavirus disease 2019 (COVID-19), por isto foram utilizadas medidas de contenção da transmissão, como o distanciamento social e o confinamento, medidas com conhecidos efeitos estressantes que podem influenciar o comportamento alimentar. Portanto, o objetivo desta pesquisa é identificar o comportamento alimentar por meio da restrição dietética, a alimentação emocional e a ingestão descontrola$\mathrm{da}$, e explorar os fatores associados a esses comportamentos em adultos durante o confinamento por COVID-19 em países de língua espanhola.

Método: será realizado um estudo transversal descritivo, por meio de um questionário online autoaplicável, que será composto por um questionário de comportamento alimentar (Three-Factor Eating Questionnaire-R18), informações 
estilo de vida antes y durante el confinamiento. Se realizará un análisis descriptivo de las variables, análisis bivariante y modelos de regresión lineal multivariantes para explorar los factores asociados con la conducta alimentaria en el distanciamiento social.

Discusión: el confinamiento ha modificado la ingesta de alimentos hacia un patrón alimentario no saludable, reducción de la actividad física e incremento del sedentarismo. Sin embargo, no se ha analizado el papel de esta medida de contención en la conducta alimentaria desde la perspectiva de restricción, emociones e ingesta descontrolada, situación que brinda la oportunidad de estudiar este fenómeno en la población hispana e identificar los posibles factores asociados que puedan sentar las bases de intervenciones durante el confinamiento o posterior a este.

Palabras clave: COVID-19, cuarentena, conducta alimentaria, protocolo. to explore the factors associated with food behavior in the pandemic confinement.

Discussion: Confinement has modified food intake, towards an unhealthy eating pattern, reduction of physical activity and increased sedentary lifestyle. However, the role of this containment measure in eating behavior from the perspective of restriction, emotions and uncontrolled eating has not been analyzed. This gives the opportunity to study this phenomenon in the Hispanic population and to identify the possible associated factors that may lay the foundations for interventions during or after confinement.

Keywords: COVID-19; Quarantine; Feeding Behavior; Protocol. sociodemográficas, de saúde e características do estilo de vida antes e durante o confinamento. Será realizada uma análise descritiva das variáveis, análise bivariada e modelos de regressão linear multivariada para explorar os fatores associados ao comportamento alimentar no distanciamento social.

Discussão: o confinamento modificou a ingestão de alimentos para um padrão alimentar não saudável, reduziu a atividade física e aumentou o estilo de vida sedentário. No entanto, o papel dessa medida de contenção no comportamento alimentar não foi analisado desde a ótica da restrição, das emoções e da alimentação descontrolada, situação que oferece a oportunidade de estudar esse fenômeno na população hispânica e identificar possíveis fatores associados que possam fundamentar intervenções durante ou após o confinamento.

Palavras-chave: COVID-19, quarentena, comportamento alimentar, protocolo.

\footnotetext{
Red de Nutrición Basada en la Evidencia, Academia Española de Nutrición y Dietética.

2 Laboratorio de Investigación Traslacional en Farmacología, Facultad de Medicina, Universidad Autónoma de San Luis Potosí, México.

3 Departamento de Nutrición, Ciencias de la Alimentación y Gastronomía, Facultad de Farmacia y Ciencias de la Alimentación, Universidad de Barcelona, Barcelona, España.

4 INSA-UB, Instituto de Investigación en Nutrición y Seguridad Alimentaria, Universidad de Barcelona, Santa Coloma de Gramenet, España.

5 Grupo de Investigación $\mathrm{M}_{3} \mathrm{O}$ (Methodology, Methods, Models and Outcomes of Health and Social Sciences). Facultad de Ciencias de la Salud y el Bienestar. Universidad de Vic, Universidad Central de Cataluña, España.

6 Departamento de Ciencias de la Salud, Facultad de Medicina, Pontificia Universidad Católica de Chile, Chile.

7 Unidad de Metabolismo y Nutrición, Departamento de Investigación. Hospital Regional de Alta Especialidad del Bajío. León, Guanajuato, México.
}

\footnotetext{
8 Instituto de Investigaciones de la Facultad de Ciencias de la Salud, Universidad Católica de Santa Fe, Argentina.

9 Departamento de Gastroenterología, Instituto Nacional de Ciencias Médicas y Nutrición Salvador Zubirán, Ciudad de México, México.

10 Nutrir México, México.

11 IIBISMED-Instituto de Investigaciones Biomédicas e Investigación Social. Facultad de Medicina, Universidad Mayor de San Simón, Cochabamba, Bolivia.

12 Grupo de Investigación Techné Ingeniería del conocimiento y del producto. Universidad de Granada, Granada, España.

13 Escuela de Nutrición, Universidad de la República, Uruguay.

14 Grupo de Investigación en Terapia Ocupacional (InTeO) de la Universidad Miguel Hernández de Elche, España.
}

*Correspondencia: Eduard Baladia.

e.baladia@rednube.net

\section{INTRODUCCIÓN}

En el transcurso de diciembre 2019 en la ciudad de Wuhan, provincia de Hubei (República Popular China), las autoridades sanitarias identificaron un grupo de casos de neumonía de etiología desconocida. Se observaron vínculos entre los casos índice y el mercado de mariscos del sur de China. Con la posibilidad de un nuevo brote de zoonosis o síndrome respiratorio agudo severo (SARS) en mente, se emprendieron investigaciones que desde entonces han identificado un nuevo coronavirus,

el SARS-CoV-2 (anteriormente, 2019-nCoV), como el agente causal de la nueva enfermedad respiratoria, coronavirus disease 2019 (COVID-19) ${ }^{(1)}$.

La Organización Mundial de la Salud (OMS) declaró la pandemia de COVID-19 el 11 de marzo de 2020, bajo los criterios y descriptores establecidos, que afectó a países de todos los continentes. Para responder a la COVID-19, muchos países han recurrido a una combinación de actividades de contención y mitigación con la intención de retrasar las grandes oleadas de casos y nivelar la demanda de camas y servicios 
médicos hospitalarios, al tiempo que protegen a la población más vulnerable a la infección, incluidas las personas mayores y las personas con comorbilidades y enfermedades crónicas ${ }^{(2)}$.

Dentro de las actividades de contención se encuentran el aislamiento de casos, cuarentena, confinamiento y distanciamiento social. El objetivo principal de estas medidas de salud pública es prevenir la propagación de la enfermedad de persona a persona ${ }^{(3)}$.

La cuarentena es la separación y restricción del movimiento de personas que potencialmente han estado expuestas a una enfermedad infectocontagiosa para determinar la presencia de sintomatología clínica $\mathrm{y}$, con ello, reducir el riesgo de transmisión ${ }^{(4)}$. Esta práctica se remonta a Venecia, Italia, en 1127 durante la pandemia de lepra, así como durante la respuesta a la peste negra ${ }^{(5)}$. La definición de cuarentena difiere del distanciamiento social, este último está diseñado para reducir las interacciones entre las personas en una comunidad más amplia, en la que los individuos pueden ser infecciosos, pero aún no han sido identificados y, por tanto, aún no se encuentran aislados ${ }^{(3)}$.

Una revisión de 24 estudios sugirió que el impacto psicológico del confinamiento es amplio y sustancial y podría ser duradero, con efectos psicológicos negativos, incluidos síntomas de estrés postraumático, confusión y enojo. Los factores estresores identificados son la duración del confinamiento, temores de infección o reinfección, frustración, aburrimiento, suministro deficiente de alimentos y consumo inadecuado de los mismos, crisis de información inapropiada, pérdidas financieras y estigma social ${ }^{(6)}$.

Como se ha descrito en la literatura, la conducta alimentaria responde a una combinación de diversos factores: biológicos, psicológicos y socioculturales ${ }^{(7)}$. Las modificaciones de estos factores pueden incidir en la ingesta de alimentos; tal es el caso de las situaciones de estrés, que pueden ser detonantes de alteraciones en la conducta alimentaria ${ }^{(8-10)}$.

Durante el actual confinamiento se han observado cambios importantes en la ingesta de alimentos, caracterizados por un patrón alimentario no saludable, mayor ingesta de productos ultraprocesados y de baja calidad nutricional ${ }^{(11)}$, así como incremento en el peso corporal, disminución de la actividad física, incremento del sedentarismo, mayor consumo de refrigerios y disminución del consumo de alimentos frescos ${ }^{(12)}$, lo que podría ser resultado de los cambios emocionales y estresores vividos durante este período. Es necesario mencionar que los efectos del confinamiento se pueden presentar en todo el espectro y continuo de la conducta alimentaria, desde la subingesta hasta la sobreingesta, y esto será mediado por diversos factores, entre ellos, el estado de nutrición y la percepción sobre la salud ${ }^{(13)}$.

Actualmente se ha documentado bien el papel del sobrepeso, la obesidad y las enfermedades crónicas relacionadas con la nutrición en el desenlace de infección por SARS-CoV-2, que incrementan el riesgo de morbimortalidad $^{(14)}$. Por esta razón, es necesario identificar el papel de las medidas de control de la pandemia por COVID-19 en la conducta alimentaria y su efecto en el estado de nutrición.

En los últimos meses se han publicado diversos estudios a nivel internacional que analizan los cambios en el patrón alimentario de la población en distanciamiento social, tal como el anteriormente citado ECLBCOVID-19 International Online Survey ${ }^{(11)}$, el análisis de dieta y actividad física en la cohorte francesa NutriNetSanté (12) $^{(12)}$ el estudio realizado en México sobre los cambios en el estilo de vida y la nutrición ${ }^{(15)}$. Sin embargo, en estos estudios no se ha incluido el análisis de la alimentación emocional, la cual podría explicar la mayoría de los cambios en el patrón y la conducta alimentaria durante el confinamiento ${ }^{(8)}$.

La alimentación emocional se refiere a la tendencia a comer en exceso en respuesta a emociones negativas como resultado de una mala conciencia interoceptiva $^{(16)}$. El papel de las emociones puede modificar de forma importante la elección, cantidad y calidad de la ingesta alimentaria ${ }^{(17)}$; los cambios emocionales que suponen el confinamiento por la pandemia por COVID-19 convierten a la alimentación emocional en un tema de creciente preocupación ya que se ha relacionado con el consumo excesivo de alimentos ricos en calorías y grasas, particularmente en situaciones de estrés $^{(18)}$, así como con mayor riesgo de obesidad ${ }^{(19)}$. En el estudio de Brooks y colaboradores se describió que las personas sometidas a largos períodos de estrés tienden a tener conductas de dependencia a sustancias, especialmente en situaciones de crisis, y estos cambios pueden perdurar incluso al término del período de estrés ${ }^{(6)}$. Además, se ha observado que la alimentación emocional se asocia significativamente con la sobrealimentación autoinformada ${ }^{(16)}$.

Ante la falta de información existente sobre la conducta alimentaria emocional en el confinamiento por COVID-19, se realizará esta investigación a partir de la siguiente pregunta: ¿Cuáles son los factores asociados con la conducta alimentaria a través de la restricción dietética, alimentación emocional e ingesta incontro- 
lada en adultos durante el confinamiento por la pandemia de la COVID-19 en países hispanohablantes?, y los objetivos generales son identificar la conducta alimentaria a través de la restricción dietética, alimentación emocional e ingesta incontrolada, y explorar los factores asociados con estas conductas en adultos durante el confinamiento por la pandemia de COVID-19 en países de habla hispana.

\section{MÉTODO}

Estudio observacional de corte transversal, mediante un cuestionario en línea autoaplicado en la plataforma Google Forms. Este protocolo de investigación sigue las guías STROBE (STrengthening the Reporting of OBservational Studies in Epidemiology) para los estudios transversales.

La población estará conformada por adultos de cualquier sexo, entre 18 y 65 años, residentes en países hispanohablantes y en situación de confinamiento obligatorio o voluntario de al menos 7 días. A través de un muestreo no probabilístico por conveniencia, se reclutará a los participantes empleando como divulgación de la encuesta las redes sociales, correos electrónicos, medios de comunicación masiva y sitios web relacionados con la nutrición de los diferentes países participantes y a través redes académicas y personales de los investigadores a cargo del presente proyecto.

El cuestionario en línea autoaplicado estará integrado por preguntas estructuradas en las siguientes secciones:

1. Cuestionario de conducta alimentaria: para la medición de la conducta alimentaria: se empleará un instrumento validado, el Three-Factor Eating Questionnaire-R18 (TFEQ-SP), el cual mide tres aspectos diferentes del comportamiento alimentario: alimentación restringida (definida como restricción consciente de la ingesta de alimentos dirigida a controlar el peso corporal o promover la pérdida de peso), comer sin control (la tendencia a comer más de lo habitual debido a una pérdida de control sobre la ingesta con una sensación subjetiva de hambre) y alimentación emocional (incapacidad para resistir las señales emocionales, comer como respuesta a diferentes emociones negativas). El cuestionario consta de 18 ítems que se miden en una escala de respuesta de 4 puntos (definitivamente cierto: 1 , mayormente verdadero: 2 , mayormente falso: 3 , definitivamente falso: 4) y los puntajes de los ítems se suman en puntajes de subescala: restricción cognitiva (RC), alimentación emocional (EE) e ingesta incontrolada (UE) ${ }^{(20)}$.
2. Información sociodemográfica y de salud (antropométricos, enfermedades crónicas y sociodemográficos): en la sección de Información sociodemográfica $y$ de salud se incluirán: datos antropométricos básicos autorreportados (peso $[\mathrm{kg}]$, altura $[\mathrm{cm}]$, índice de masa corporal [IMC, estimado], circunferencia abdominal $[\mathrm{cm}])$, información sobre el estado de salud (diagnóstico previo de enfermedades crónicas: diabetes, hipertensión, cáncer, obesidad; y tiempo transcurrido desde el diagnóstico de esos padecimientos) y datos sociodemográficos (país de residencia, sexo [masculino/femenino], edad, nivel educativo [primarios, secundarios, universitarios], distinción de profesional sanitario, situación laboral previa a la pandemia y actual [trabaja, teletrabajo, jubilado pensionista, parado/cobrando subsidio, parado/sin cobrar, estudiante, trabajo doméstico no remunerado, u otras situaciones], estado civil, número de convivientes e ingresos familiares).

3. Estilo de vida (distanciamiento, estilo de vida y adquisición de alimentos): la última sección indagará sobre el estilo de vida: días de confinamiento que lleva el individuo, tipo de convivencia (solo/a, en familia sin hijos, en familia con hijos), frecuencia y motivo de salidas fuera de la casa; datos relacionados con estilo de vida antes y durante el confinamiento por la pandemia: calidad del sueño, actividad física, uso de pantallas, hábito tabáquico (nunca fumador; exfumador; fumador); y datos relacionados con la compra de alimentos: frecuencia y gasto monetario en la compra de alimentos en la última semana, frecuencia de compra de comida a domicilio.

\section{ANÁLISIS ESTADÍSTICO}

Se realizará un análisis descriptivo de las variables, las de escala continua se presentarán como medias y desviación típica o medianas y rangos intercuartílicos de acuerdo con la prueba de normalidad de Kolmogórov-Smirnov.

Para los análisis bivariantes entre las tres variables de conducta alimentaria según las variables sociodemográficas y de estilos de vida se utilizarán la media o la mediana, así como la desviación típica y el rango intercuartílico para describir las conductas según tengan una distribución normal o no. Para calcular el valor $p$ de comparación se utilizará la prueba t Student o ANOVA si siguen una distribución normal, o U de Mann-Whitney o Kruskall Wallis si no sigue una distribución normal, dependiendo del número categorías de las variables sociodemográficas o de estilos de vida. 
Para explorar los factores asociados con una mayor puntuación en las variables de conducta alimentaria se utilizarán modelos de regresión lineal multivariantes clásicos o robustos por el método de mínimos cuadrados, dependiendo de si se cumplen o no las hipótesis del modelo de regresión. Todos los análisis se realizarán con el software estadístico libre R y se establecerán contrastes bilaterales y con un nivel de significación de 0,05.

\section{ASPECTOS ÉTICOS}

El presente protocolo de investigación fue presentado en Open Science Framework (https://osf.io/dz9s7/), que permitió una preevaluación por expertos en el área y en la bioética, apegándose a los lineamientos éticos en investigación en seres humanos, establecidos en la Declaración de Helsinki de 1964, así como con las elaboradas por el Consejo de Organizaciones Internacionales de Ciencias Médicas (CIOMS) en colaboración con la OMS para la investigación en salud durante desastres, emergencias o brotes epidémicos (CIOM, Guía 20), y por su naturaleza se garantizará un estudio de riesgo mínimo y se respetará el otorgamiento del consentimiento informado por cada persona de investigación.

Al tratarse de un estudio observacional y transversal, implica riesgo mínimo para los participantes, debido a que no se hará intervención alguna. Aunque los datos se obtendrán a partir de cuestionarios realizados mediante dispositivos electrónicos y a través de internet, los investigadores se comprometen a establecer los mecanismos necesarios para salvaguardar los datos personales de cada participante, toda vez que no se va a publicar información sensible que pueda identificarles. Además, atendiendo al principio de autonomía, se pedirá, también vía virtual, el consentimiento informado de participación.

\section{DISCUSIÓN}

El objetivo de este protocolo de investigación es identificar la conducta alimentaria a través de la restricción dietética, alimentación emocional e ingesta incontrolada, y explorar los factores asociados (socioeconómicos, de salud, actividad física y sedentarismo) con estas conductas en adultos durante la pandemia por COVID-19 en países de habla hispana.

Aunque el confinamiento y distanciamiento social representa una situación de gran interés para la salud pública, y desde el campo de la nutrición y dietética, se pretende conocer el impacto que tiene esta medida en la selección, el procesamiento y la ingesta de alimentos, así como en el estilo de vida y en la seguridad alimentaria, con el objetivo de responder a las necesidades que se identifiquen en la población.

En estudios previamente publicados, se han reportado cambios en la ingesta de alimentos durante el confinamiento y se observaron patrones alimentarios no saludables ${ }^{(11,12)}$, tales como el incremento en el consumo de refrigerios ${ }^{(21)}$, consumo de alimentos con alto índice glucémico ${ }^{(22)}$ y mayor ingesta de alimentos ultraprocesados ${ }^{(23)}$. Estos cambios se han observado en todos los grupos etarios, $\mathrm{y}$ ha sido mayormente visible en sectores con desigualdades sociales importantes. No solo la ingesta de alimentos se ha visto modificada, sino también se ha reportado un incremento en el consumo de alcohol y tabaco ${ }^{(24)}$.

Otro efecto del confinamiento por la pandemia ha sido la disminución de la actividad física en la población y se ha incrementado de forma importante el comportamiento sedentario, debido a las características del teletrabajo o teleeducación ${ }^{(23)}$. En este estudio, la actividad física se aborda como un factor asociado con la conducta alimentaria a partir del análisis indirecto de los cambios en la realización previa y durante el confinamiento, así como el tiempo dedicado a las pantallas o dispositivos electrónicos.

Como resultado de estas modificaciones en la ingesta de alimentos y el estilo de vida, se ha descrito que durante el confinamiento la población ha incrementado el peso corporal ${ }^{(25-27)}$, dicho proceso ha sido mediado en alguna forma por el estado conductual de la población $^{(28,29)}$. Más del $50 \%$ de la población ha reportado datos de depresión, ansiedad, estrés e irritabilidad, y esta sintomatología está asociada con la nutrición, los hábitos del sueño y de actividad física ${ }^{(24)}$. Es importante mencionar que esta situación se incrementa en las mujeres y en el grupo de edad entre 18 y 34 años $^{(30)}$.

Es así que se fundamenta la necesidad de estudiar la conducta alimentaria, más allá de la ingesta de alimentos y su preparación, de modo que se integren los cambios conductuales y emocionales que se viven durante el confinamiento, situaciones que pueden modificar en mayor o menor medida la ingesta de alimentos y el estado nutricional ${ }^{(31,32)}$. Sin embargo, esta situación no ha sido abordada en los países de habla hispana, lo que representa la oportunidad de incluir en el análisis los posibles factores asociados con la conducta alimentaria desde la perspectiva de la restricción, el consumo emocional y la ingesta incontrolada. El estudio de este fenómeno permitirá identificar las diferencias entre países 
y los factores relacionados con las modificaciones en la conducta alimentaria durante el confinamiento, con el fin de dar respuesta a las necesidades de la población y, de este modo, fundamentar el diseño de estrategias de contención de los cambios nutricionales, conductuales y de estilo de vida que puedan incrementar la morbimortalidad por COVID-19.

A pesar de identificarse las oportunidades de este proyecto, es necesario plantear los sesgos potenciales asociados con el diseño metodológico de este protocolo. Los posibles sesgos serían de voluntario, de selección, de autorreporte y de desgaste. Estos pueden conducir a errores como información proveniente de población interesada en temas de salud y subestimación/sobreestimación de datos antropométricos y sociodemográficos, así como la no respuesta de la encuesta por la población. Se espera que la participación de un mayor número de personas de investigación redistribuya de forma similar estos sesgos y no afecten los resultados a presentarse.

\section{Fuentes de financiamiento}

El presente estudio no tuvo financiamiento.

\section{Conflicto de interés}

Los autores declaran no tener conflictos de interés que afecten la redacción de este manuscrito ni la realización de este proyecto de investigación.

\section{Contribución de autores}

R. A-P participó en la redacción del artículo y la concepción del protocolo de investigación, E. B. participó en la redacción del artículo y la concepción del protocolo de investigación, C. R-C. participó en la redacción del artículo, R. R-C. participó en la redacción del artículo, A. V-M. participó en la redacción del artículo y la concepción del protocolo de investigación, J. M. O. participó en la concepción del protocolo de investigación, E. A-J. participó en la redacción del artículo y la concepción del protocolo de investigación, A. L-L. participó en la concepción del protocolo de investigación, K. B-B. participó en la concepción del protocolo de investigación, M. C. N. participó en la redacción del artículo y la concepción del protocolo de investigación, S. E. M-V. participó en la redacción del artículo y la concepción del protocolo de investigación, S. C-L. participó en la redacción del artículo y la concepción del protocolo de investigación, A. Z-V. participó en la concepción del protocolo de investigación, P. M-L. participó en la redacción del artículo y la concepción del protocolo de investigación, L. M. R. participó en la redacción del artículo y la concepción del protocolo de investigación, E. M. N-M. participó en la redacción del artículo y la concepción del protocolo de investigación. Todos los autores revisaron el artículo y validaron su versión final.

\section{Referencias bibliográficas}

1. Lake MA. What we know so far: COVID-19 current clinical knowledge and research. Clin Med. 2020;20(2):124-27. doi: 10.7861/clinmed.2019-coron

2. Bedford J, Enria D, Giesecke J, Heymann DL, Ihekweazu C, Kobinger G, et al. COVID-19: towards controlling of a pandemic. Lancet. 2020;395(10229):1015-18. doi: 10.1016/ S0140-6736(20)30673-5

3. Wilder-Smith A, Freedman DO. Isolation, quarantine, social distancing and community containment: pivotal role for oldstyle public health measures in the novel coronavirus (2019nCoV) outbreak. J Travel Med. 2020;27(2):taaa020. doi: $10.1093 / \mathrm{jtm} /$ taaa020

4. Quarantine and Isolation [Internet]. Centers for Disease Control and Prevention; 2017 [consultado el 29 de diciembre de 2020]. Disponible en: https://www.cdc.gov/quarantine/ index.html

5. Newman KLS. Shutt up: bubonic plague and quarantine in early modern England. J Soc Hist. 2012;45(3):809-34. doi: $10.1093 /$ jsh/shr114

6. Brooks SK, Webster RK, Smith LE, Woodland L, Wessely $\mathrm{S}$, Greenberg N, et al. The psychological impact of quarantine and how to reduce it: rapid review of the evidence. Lancet. 2020;395(10227):912-20. doi: 10.1016/S01406736(20)30460-8

7. Shepherd R. Social determinants of food choice. Proc Nutr Soc. 1999;58(4):807-12. doi: 10.1017/S0029665199001093

8. Casper RC. Depression and eating disorders. Depress Anxiety. 1998;8 Suppl 1:96-104. doi: 10.1002/(SICI)15206394(1998)8:1+<96::AID-DA15>3.0.CO;2-4

9. Meyer BJ, Kolanu N, Griffiths DA, Grounds B, Howe PRC, Kreis IA. Food groups and fatty acids associated with selfreported depression: an analysis from the Australian National Nutrition and Health Surveys. Nutrition. 2013;29(7-8):10427. doi: 10.1016/j.nut.2013.02.006

10. Ramón AE, Martínez AB, Granada LJM, Echániz SE, Pellicer GB, Juárez VR, et al. Conducta alimentaria y su relación con el estrés, la ansiedad, la depresión y el insomnio en estudiantes universitarios. Nutr Hosp. 2019;36(6):1339-45. doi: 10.20960/nh.02641

11. Ammar A, Brach M, Trabelsi K, Chtourou H, Boukhris O, Masmoudi L, et al. Effects of COVID-19 home confinement on eating behaviour and physical activity: results of 
the ECLB-COVID19 International online survey. Nutrients. 2020;12(6):1583. doi: 10.3390/nu12061583

12. Deschasaux-Tanguy M, Druesne-Pecollo N, Esseddik Y, Szabo de Edelenyi F, Allès B, Andreeva VA, et al. Diet and physical activity during the COVID-19 lockdown period (March-May 2020): results from the French NutriNet-Sante cohort study. medRxiv. 2020:1-21. doi: 10.1101/2020.06.04.20121855

13. Hebden L, Chan HN, Loulie JC, Rangan A, Allman-Farinelli $\mathrm{M}$. You are what you choose to eat: factors influencing young adults' food selection behavior. J Hum Nutr Diet. 2015;28(4):401-8. doi: 10.1111/jhn.12312

14. Földi M, Farkas N, Kiss S, Zádori N, Váncsa S, Szakó L, et al. Obesity is a risk factor for developing critical condition in COVID-19 patients: A systematic review and meta-analysis. Obes Rev. 2020;21(10):e13095. doi: 10.1111/obr.13095

15. Villaseñor LK, Jimenez GAM, Ortega RAE, Islas RLM, Gonzalez MOA, Silva PTS. Cambios en el estilo de vida y nutrición durante el confinamiento por SARS-CoV-2 (COVID19) en México: Un estudio observacional. Rev Esp Nutr Hum Diet. 2021;25(Supl 2):1099.

16. Van Strien T, Herman CP, Verheijden MW. Eating style, overeating, and overweight in a representative Dutch sample. Does external eating play a role? Appetite. 2009;52(2):380-7. doi: 10.1016/j.appet.2008.11.010

17. Palomino-Pérez AM. Rol de la emoción en la conducta alimentaria. Rev Chil Nutr. 2020;47(2):289-91. doi: 10.4067/ S0717-75182020000200286

18. Oliver G, Wardle J, Gibson EL. Stress and food choice: a laboratory study. Psychosom Med. 2000;62(6):853-65. doi: 10.1097/00006842-200011000-00016

19. Sung J, Lee K, Song Y-M, Lee MK, Lee, D-H. Heritability of eating behavior assessed using the DEBQ (Dutch Eating Behavior Questionnaire) and weight-related traits: the Healthy Twin Study. Obesity. 2010;18(5):1000-5. doi: 10.1038/oby.2009.389

20. Anglé S, Engblom J, Eriksson T, Kautiainen S, Saha M-T, Lindfors $\mathrm{P}$, et al. Three factor eating questionnaire-R18 as a measure of cognitive restraint, uncontrolled eating and emotional eating in a sample of young Finnish females. Int J Behav Nutr Phys Act. 2009;6:41. doi: 10.1186/1479-5868-6-41

21. Blaszczyk-Bebenek E, Jagielski P, Boleslawska I, Jagielska A, Nitsch-Osuch A, Kawalwc P. Nutrition behaviors in Polish adults before and during COVID-19 lockdown. Nutrients. 2020;12(10):3084. doi: 10.3390/nu12103084

22. Zupo R, Castellana F, Sardone R, Sila A, Giagulli VA, Triggiani $\mathrm{V}$, et al. Preliminary trajectories in dietary behaviors during the COVID-19 pandemic: A public health call to action to face obesity. Int J Environ Res Public Health. 2020;17(19):7073. doi: 10.3390/ijerph17197073
23. Ruiz-Roso MB, de Carvalho PP, Matilla-Escalante DC, Brun P, Ulloa N, Acevedo-Correa D, et al. Changes of physical activity and ultra-processed food consumption in adolescents from different countries during Covid-19 pandemic: An observational study. Nutrients. 2020;12(8):2289. doi: $10.3390 /$ nu 12082289

24. Rossinot H, Fantin R, Venne J. Behavioral changes during COVID-19 confinement in France: a web-based study. Int J Environ Res Public Health. 2020;17(22):8444. doi: 10.3390/ ijerph17228444

25. Reyes-Olavarría D, Latorre-Román PA, Guzmán-Guzmán IP, Jerez-Mayorga D, Caamaño-Navarrete F, Delgado-Floody P. Positive and negative changes in food habits, physical activity patterns, and weight status during COVID-19 confinement: associated factors in the Chilean population. Int J Environ Res Public Health. 2020;17(15):5431. doi: 10.3390/ ijerph17155431

26. López-Moreno M, Iglesias LMT, Miguel M, Garcés-Rimón M. Physical and psychological effects related to food habits and lifestyle changes derived from COVID-19 home confinement in the Spanish population. Nutrients. 2020;12(11):3445. doi: $10.3390 /$ nu 12113445

27. Kriaucioniene V, Bagdonavicine L, Rodriguez-Perez C, Petkeviciene J. Associations between changes in health behaviours and body weight during the COVID-19 quarantine in Lithuania: The Lithuanian COVIDiet Study. Nutrients. 2020;12(10):3119. doi: 10.3390/nu12103119

28. Fernández-Río J, Cecchini JA, Mendez-Gimenez A, Carriedo A. Weight changes during the COVID-19 home confinement. Effects on psychosocial variables. Obes Res Clin Pract. 2020;14(4):383-85. doi: 10.1016/j.orcp.2020.07.006

29. Zachary Z, Forbes B, Lopez B, Pedersen G, Welty J, Deyo A, et al. Self-quarantine and weight gain related risk factors during the COVID-19 pandemic. Obes Res Clin Pract. 2020;14(3):210-16. doi: 10.1016/j.orcp.2020.05.004

30. Antunes R, Frontini R, Amaro N, Salvador R, Matos R, Morouço P, et al. Exploring lifestyle habits, physical activity, anxiety and basic psychological needs in a sample of Portuguese adults during COVID-19. Int J Environ Res Public Health. 2020;17(12):4360. doi: 10.3390/ijerph17124360

31. Shen W, Long LM, Shih C-H, Ludy M-J. A humanities-based explanation for the effects of emotional eating and perceived stress on food choice motives during the COVID-19 pandemic. Nutrients. 2020;12(9):2712. doi: 10.3390/nu12092712

32. Al-Musharaf S. Prevalence and predictors of emotional eating among healthy young Saudi women during the COVID-19 pandemic. Nutrients. 2020;12(10):2923. doi: 10.3390/ nu12102923 